\section{Rapport annuel 2000 Groupe FMH Services}

\author{
Markus Baumgartner, directeur de FMH Services
}

L'office de placement a également enregistré une hausse du nombre des demandes d'emploi de la part des médecins-assistants et des assistantes médicales.

Le réseau des fiduciaires regroupées dans FMH Services Treuhand n'a pas été étendu en l'an 2000. Les succursales de Muri, Olten, Oberuzwil, Stans, Zurich, Coire, Echallens, Sierre, Genève et Chiasso n'ont cependant pas eu à se plaindre du manque de mandats.

En ce qui concerne la planification et l'aménagement des cabinets médicaux, nous avons à nouveau pu compter sur la précieuse collaboration de Aefa Design à Berne et de Promedical SA à Yverdon-lesBains.

La pression exercée par la Confédération en matière d'assurance-qualité dans le domaine de la radiologie s'est faite sentir l'année dernière chez les médecins de premier recours mais, grâce à l'entreprise Wiroma et ses conseils pour toutes questions ayant trait à la radiologie, de nombreux problèmes ont pu être résolus de manière satisfaisante.

L'assemblée générale a approuvé le 30 juin 2001, selon la proposition de l'organe de révision, le rapport annuel et les comptes pour l'année 2000, ainsi que le budget pour 2001 .

\section{Contact}

FMH Consulting Services, Burghöhe 1, case postale 246, 6208 Oberkirch, tél. 04192500 77, fax 041 92105 86, e-mail: mail@fmhconsulting.ch

\section{FMH Inkasso Services}

Direction: René Häller

Collaborateurs: 19 personnes (dont 13 à temps partiel)

FMH Inkasso Services, dont les bureaux se trouvent à Muri (BE) et à Genève ont traité plus de 22500 dossiers de débiteurs lors de l'exercice comptable 1999 (d'un montant total de plus de 10 millions de francs). Grâce à une méthode de travail individualisée qui tient compte de la relation entre patient et médecin, le taux de succès a également été élevé lors de l'exercice écoulé et les mandants n'ont ainsi pas eu à subir de fâcheuses pertes d'honoraires.

FMH Inkasso Services, en tant que filiale à part entière de l'organisation de prestations à but non lucratif qu'est FMH Services, est tenue de respecter la philosophie d'entreprise de cette dernière. Etant donné qu'aucune taxe annuelle de base n'est exigée des mandants, l'attribution de mandats annuels présente des avantages.

L'assemblée générale a approuvé le 30 juin 2001, selon la proposition de l'organe de révision, le rapport annuel et les comptes pour l'année 2000, ainsi que le budget pour 2001 .

\section{Contact}

FMH Inkasso Services, Thorackerstrasse 3, 3074 Muri, tél. 03135912 10, fax 03135912 00, e-mail: mail@fmhinkasso.ch 


\section{FMH Insurance \&t Financial Services}

Direction: Peter Gygax, Thomas Roth

Collaborateurs: 3 personnes (à temps partiel)

FMH Assurance Services existe depuis le milieu de l'année 1999. Cette société a un nouveau nom depuis la fin 2000 en raison de l'extension des prestations dans le domaine de l'assurance et des finances. Elle s'appelle désormais «FMH Insurance \&t Financial Services".

Outre l'offre déjà connue pour le domaine des caisses-maladie, toutes les autres assurances et placements financiers seront aussi proposés.

En raison de sa nouvelle collaboration avec la firme Krachpelz SA de Berne, spécialisée dans les questions relatives aux assurances et aux capitaux, FMH Insurance \& Financial Services SA est désormais indépendante. Cette indépendance est un plus pour la société. A l'heure actuelle, les prestations d'assurance et les prestations financières comprennent tellement de produits qu'il est difficile pour un néophyte de trouver l'offre la mieux adaptée à ses besoins. Chez FMH Insurance \&t Financial Services, nous avons une vue d'ensemble et sommes donc en mesure de proposer des conseils personnalisés et des produits appropriés. Cela concerne notamment les questions de financement et d'assurance lors de l'ouverture d'un cabinet médical, ainsi que celles concernant les assurances et la prévoyance professionnelle du détenteur du cabinet médical et de ses employés. FMH Insurance \& Financial Services fournit également de très bons conseils en ce qui concerne les assurances de capitaux et les assurances risques, les assurances de choses et les assurances responsabilité civile.

L'assemblée générale de FMH Assurance Services a approuvé le 30 juin 2001, selon la proposition de l'organe de révision, le rapport annuel et les comptes pour l'année 2000, ainsi que le budget pour 2001.

\section{Contact}

FMH Insurance \& Financial Services, Mattenstrasse 11, 3073 Gümligen, tél. 03195950 00, fax 031 95950 10, e-mail: mail@fmhinsurance.ch

\section{FMH Congress \& Travel Services}

\section{Direction: Markus Baumgartner}

Collaborateurs: 2 personnes (à temps partiel)

FMH Congress \&t Travel a, durant l'exercice écoulée, a été très occupée par les travaux préparatoires en vue du $1^{\text {er }}$ congrès de Managed Health Care qui s'est déroulé en avril 2001 à Winterthour. Le deuxième congrès aura lieu en 2002. Les travaux préparatoires sont déjà en cours.
Malheureusement, les démarches entreprises pour obtenir des offres de voyage plus avantageuses pour les membres de la FMH se sont avérées vaines. De nombreux pourparlers ont été engagés avec des agences de voyages, mais il semblerait que les voyagistes ne soient guère intéressés par une collaboration avec les quelque 28000 membres de la FMH et leurs familles. La direction a donc décidé de se concentrer principalement sur l'organisation de congrès et de proposer un ou deux voyages d'études par an, consacrés à des thèmes spécifiques au domaine de la santé.

L'assemblée générale a approuvé le 30 juin 2001, selon la proposition de l'organe de révision, le rapport annuel et les comptes pour l'année 2000, ainsi que le budget pour 2001.

\section{Contact}

FMH Congress \& Travel Services, Burghöhe 1, case postale 246, 6208 Oberkirch, tél. 04192500 77, fax 04192105 86, e-mail@fmhcongress.ch

\section{FMH Marketing \& Kommunikation}

Direction: Lahor Jakrlin

Collaborateurs: 2 (occupation à 20\% au plus selon mandats)

FMH Marketing \& Kommunikation est une agence de publicité créée pour répondre aux besoins du corps médical en la matière. Cette société travaille exclusivement avec des agences renommées telles que Fruitcake, MCH et F. Mosimann. Les agences de publicité Fruitcake ont des succursales à Worb, Lucerne et Zurich. MCH a son siège social à Winterthour.

Outre les offres traditionnelles de marketing et de publicité telle que la conception de brochures, la création de campagnes de publicité et l'organisation de manifestations de relations publiques, la société FMH Marketing \& Kommunikation prodigue également des conseils dans le domaine de la communication. De nombreux médecins et hôpitaux ont fait appel à cette société au cours de l'exercice écoulé.

FMH Marketing \& Kommunikation met ses moyens publicitaires à la disposition de la FMH et de FMH Services. En effet, cette agence est l'auteur de la brochure de présentation de la FMH intitulée «Protection, Puissance, Prestations», sortie à fin 2000. Elle a en outre conçu et réalisé dans un très bref délai le stand de la FMH pour le congrès «Medico Vadis».

L'assemblée générale a approuvé le 30 juin 2001, selon la proposition de l'organe de révision, le rapport annuel et les comptes pour l'année 2000, ainsi que le budget pour 2001.

\section{Contact}

FMH Marketing \& Kommunikation, Burghöhe 1, case postale 246, 6208 Oberkirch, tél. 04192500 77, fax 04192105 86, e-mail: mail@fmhservices.ch 